(ii) The intersection of the closed exteriors of the circles of curvature of $C$, and the intersection of the closed exteriors of the minimal circumscribed circles to $C$.

\title{
REFERENCE
}

1. W. Blaschke, Vorlesungen über Differentialgeometrie I, Berlin, 1930.

University of California at Santa Barbara and Los Angeles and INSTITUTE FOR ADVANCED STUdY

\section{HOMOTOPY GROUPS OF ONE-DIMENSIONAL SPACES}

\author{
M. L. CURTIS ${ }^{1}$ AND M. K. FORT, JR.
}

In this paper we prove the following theorem:

If $S$ is a one-dimensional separable metric space, then $\pi_{k}(S)=0$ for all $k>1$.

Actually it is proved that a much broader class of spaces than spheres have the property that mappings of these spaces into onedimensional spaces are homotopic to constant maps. This class of spaces includes, for example, projective spaces and Lens spaces.

Lemma $1 .^{2}$ Let $X$ be a compact metric space whose one-dimensional integral singular homology group is a torsion group. Then for any finite covering $G$ of order one by arcwise-connected open sets, $G$ does not contain a simple loop.

Proof. By a simple loop we mean a simple chain such that the first and last sets are the same. Let $K$ be the nerve of $G$. Since $K$ is onedimensional, a simple loop in $G$ implies a nonbounding one cycle in $K$. Hence it suffices to show that $H_{1}(K)=0$.

Let $\phi: X \rightarrow K$ be a canonical map. For each vertex $v$ in $K$ we choose a point $\psi(v)$ in the element of $G$ corresponding to $v$. For each edge $\sigma$ with vertices $v_{1}$ and $v_{2}$ we extend $\psi$ on $\left\{v_{1}, v_{2}\right\}$ to a mapping of $\sigma$ into the union of the two elements of $G$ corresponding to $v_{1}$ and $v_{2}$. This is possible, since these two elements of $G$ are arcwise connected and

Received by the editors August 22, 1956 and, in revised form, September 28, 1956.

1 Supported by contract AF 18(600)-1571.

2 The method of proof used below, which extended this lemma from manifolds $M$ with $H_{1}(M)=0$ to compact spaces $X$ with $H_{1}(X)$ a torsion group, was suggested by the referee. 
must have a non-null intersection by the definition of the nerve of a covering. This defines a map $\psi: K \rightarrow X$, and it is easy to check that the map $\phi \psi: K \rightarrow K$ is star related with the identity so that $\phi \psi$ is homotopic to the identity map. It follows from this that $\psi_{*}: H_{1}(K)$ $\rightarrow H_{1}(X)$ is an isomorphism into. Since $H_{1}(X)$ is a torsion group, so is $H_{1}(K)$. Since $K$ is a graph, $H_{1}(K)$ is free abelian, so that $H_{1}(K)=0$. This proves the lemma.

Lemma 2. If $X$ is also a locally connected continuum, $Y=f(X)$ is onedimensional and $f$ is monotone, then $Y$ is a dendrite.

Proof. A dendrite is a locally confected continuum which does not contain a simple closed curve. We suppose that $Y$ contains a simple closed curve $\Gamma$ and obtain a contradiction.

There exists a positive number $\epsilon$ such that from any covering of $\Gamma$ by open sets of diameter less than $\epsilon$ one can extract a simple loop of open sets (which may not cover $\Gamma$ ). There exists a covering $\mathcal{u}$ of $Y$ of order one by open sets of diameter less than $\epsilon$. The set of all components of members of $\mathcal{u}$ has a finite subset $\vartheta$ which is a covering of $Y$ of order one by connected open sets of diameter less than $\epsilon$. It follows that $v$ contains a simple loop $V_{1}, \cdots, V_{n-1}, V_{1}$.

The covering $G=\left\{f^{-1}(V) \mid V \in V\right\}$ is a covering of $X$ of order one by connected open sets, and it contains a simple loop. By Lemma 1 this is not possible and Lemma 2 is proved.

THEOREM. Let $X$ be a locally connected continuum whose one-dimensional integral singular homology group is a torsion group. Let $S$ be a one-dimensional separable metric space. Then any map $f: X \rightarrow S$ is homotopic to a constant map.

Proof. Let $f=g h$ be the monotone-light factorization of $f$, and let $Y=h(X)$. Since $g$ is light, $Y$ must be one-dimensional [3, p. 91]. By Lemma 2, $Y$ is a dendrite and one of the authors [2] has shown that a dendrite is contractible. Hence $h$ is homotopic to a constant and so is $f$. This proves the theorem.

Corollary. If $S$ is a one-dimensional separable metric space, then $\pi_{k}(S)=0$ for $k>1$.

REMARK. If an arcwise connected one-dimensional separable metric space $X$ has $\pi_{1}(X)$ a free finitely-generated group, then all singular homology groups $H_{k}(X, Z)=0, k>1$. This follows because $X$ is aspherical and an aspherical space with the same fundamental group is obtained by taking a finite number of circles with one common 
point. Since the homology groups of this model are trivial in dimensions higher than one, the same is true of $X$ (see $[1$, p. 481]).

REMARK. For $k=2$ this corollary can be proved without the theorem since a monotone image of the 2-sphere is a cactoid [4] which is either a dendrite or contains a 2-sphere. This also shows that if $\pi_{2}(S) \neq 0$, then there exists a light map of $S^{2}$ into $S$. However, there exists a space $S$ for which $\pi_{2}(S) \neq 0$ such that any light map of $S^{2}$ into $S$ is inessential.

Problem. ${ }^{3}$ Is every monotone image of $S^{k}, k>1$, simply connected? For $k=2$ the answer is "yes" by the theorem of R. L. Moore [4], but we do not know the answer for $k=3$.

\section{BIBLIOGRAPHY}

1. S. Eilenberg and S. MacLane, Relations between homology and homotopy groups of spaces, Ann. of Math. vol. 46 (1945) pp. 480-509.

2. M. K. Fort, Jr. Mappings on $S^{1}$ into one-dimensional spaces, submitted to Illinois Journal of Mathematics.

3. W. Hurewicz and H. Wallman, Dimension theory, Princeton, 1941.

4. R. L. Moore, Concerning upper semi-continuous collections of continua, Trans. Amer. Math. Soc. vol. 27 (1925) pp. 416-428.

UNIVERSITY OF GEORGIA

Added in proof. This problem has been settled in the negative by R. H. Bing for $k=3$. 